\title{
A NOTE ON THE BOTT VANISHING THEOREM
}

\author{
IRA MOSKOWITZ
}

\begin{abstract}
We give a simple example of a foliated 4-manifold that shows that the bound in Bott's theorem is best possible.
\end{abstract}

Conventions. The following hold unless stated otherwise:

(1) All manifolds are closed, oriented, and $C^{\infty}$.

(2) All $H^{*}$ are with R-coefficients.

1. Introduction. Let $M^{n}$ be an $n$-manifold with a foliation $\mathscr{F}^{q}$ of codimension $q$. Let $\nu(\mathscr{F})$ denote the normal bundle of $\mathscr{F}$ and $\operatorname{Pont}^{*}(\nu(\mathscr{F}))$ the Pontrjagin algebra of $\nu(\mathscr{F})$.

(1.1) The Bott Vanishing Theorem. Pont ${ }^{i}(\nu(\mathscr{F}))=0$ for $i>2 . q$.

This very powerful theorem gives a necessary condition for a subbundle of $T M$, the tangent bundle of $M$, to be the tangent bundle $T \mathscr{F}$ of some foliation $\mathscr{F}$. In searching the literature this author was unable to find any example that showed that (1.1) was best possible in terms of dimension. However, in [T1] Thurston proved the existence of such a foliation.

(1.2) Problem. Find $\left(M^{n}, \mathscr{F}^{q}\right)$ such that $\operatorname{Pont}^{2 q}(\nu(\mathscr{F})) \neq 0$.

No claims of uniqueness are made for the example in this paper-just simplicity. A powerful theorem of Thurston is used in constructing the example.

(1.3) Theorem (T2, Corollary 3). Every 2-plane field on $M^{n}, n>3$, is homotopic to a completely integrable $C^{\infty}$-plane field.

In other words, we can always view a 2-dimensional subbundle of $T M^{n}, n>3$, as the tangent bundle of a foliation (up to bundle isomorphism).

We will construct a 4-manifold $M^{4}$ with a 2 -frame field, which certainly gives us a trivial 2-plane field. By (1.3) we can take this plane field as $T\left(\mathscr{F}^{2}\right) \cong \varepsilon^{2}$. Since $T\left(\mathscr{F}^{2}\right) \oplus \nu\left(\mathscr{F}^{2}\right) \cong T M^{4}$ we have that $p_{1}(\nu(\mathscr{F}))=p_{1}(M)$. If we can show that $p_{1}(M) \neq 0$ then we will have shown that $\operatorname{Pont}^{4}(\nu(\mathscr{F})) \neq 0$, which solves (1.2).

Received by the editors June 3, 1984.

1980 Mathematics Subject Classification. Primary 57R30, 57R25.

(1985 American Mathematical Society $0002-9939 / 85 \$ 1.00+\$ .25$ per page 
2. Example. In this section all manifolds are 4-dimensional.

Let $\mathscr{S}$ be the nonsingular bilinear pairing from

$$
H^{2}(M ; \mathbf{Z}) / \text { torsion } \otimes H^{2}(M ; \mathbf{Z}) / \text { torsion } \rightarrow \mathbf{Z}
$$

coming from Poincaré duality. We say that $\omega \in H^{2}(M ; \mathbf{Z}) /$ torsion is a $W u$ class if $\mathscr{S}(\omega, x) \equiv \mathscr{S}(x, x) \bmod 2$, for all $x \in H^{2}(M ; \mathbf{Z}) /$ torsion. Let $\Omega$ be the set $\{\mathscr{S}(\omega, \omega)\} \subset \mathbf{Z}$.

The following result of Hirzebruch and Hopf is found in [Th, Theorem 19].

(2.1) Proposition. $M^{4}$ admits a 2-frame field with finite singularities. The following pairs of integers, and only these, occur as the index for such a 2-frame field:

$$
a=\frac{1}{4}(\alpha-3 \sigma-2 \chi), \quad b=\frac{1}{4}(\alpha-3 \sigma+2 \chi),
$$

where $\sigma$ is the signature of $M^{4}, \chi$ is the Euler characteristic, and $\alpha \in \Omega$.

We will now construct a specific 4-manifold. Let

$$
\tilde{M}^{4}=\mathrm{C} P^{2} \# \mathbf{C} P^{2} \# \mathrm{C} P^{2} \# \mathbf{C} P^{2} \# T^{4} \# T^{4} \# T^{4} \text {, }
$$

where \# denotes the connected sum and $T^{4}$ is the 4-torus. Easy calculations show that $\chi\left(\tilde{M}^{4}\right)=0$, and $\sigma\left(\tilde{M}^{4}\right)=4$. Hence, our obstruction to a 2-frame field is $\frac{1}{4}(\alpha-12)$. Noting that $\mathscr{S}$ treats the connected sum operation like disjoint union, simple linear algebra shows that we may choose $\alpha=12$. Therefore, $\tilde{M}^{4}$ has a 2-frame field, and we are done since $p_{1}\left(\tilde{M}^{4}\right) \neq 0$.

ACKNOWLedgement. I wish to thank Al Sato for his assistance.

\section{REFERENCES}

[T1] W. Thurston, Foliations and groups of diffeomorphisms, Bull. Amer. Math. Soc. 80 (1974), 304-307.

[T2] _ The theory of foliations of codimension greater than one, Comment. Math. Helv. 49(1974), 214-231.

[Th] E. Thomas, Vector fields on manifolds, Bull. Amer. Math. Soc. 75 (1969), 643-683.

Department of Mathematics, Texas A \& M University, College Station, Texas 77843

Current address: Center for Naval Analyses, P. O. Box 16268, Alexandria, Virginia 22302 